\title{
Physicomechanical Properties of Nanobiocomposite Composed of Polylactic Acid and Biogenic Nano hydroxyapatite
}

\author{
N. Bano ${ }^{1,3}$, S.S. Jikan ${ }^{1 *}$, H. Basri ${ }^{1}$, S. Adzila² and N.A. Badarulzaman ${ }^{2}$ \\ ${ }^{1}$ Faculty of Applied Sciences and Technology, Universiti Tun Hussein Onn Malaysia (UTHM), 84600 Pagoh, Johor, \\ MALAYSIA.
}

\begin{abstract}
${ }^{2}$ Faculty of Mechanical and Manufacturing Engineering, Universiti Tun Hussein Onn Malaysia (UTHM), 86400 Parit Raja, Batu Pahat, Johor, MALAYSIA.
\end{abstract}

${ }^{3}$ Department of Chemistry, Government Postgraduate College for Women Raiwind, Lahore, 5400 PAKISTAN.

*Corresponding Author

DOI: https://doi.org/10.30880/ijie.2019.11.01.028

Received 24 October 2018; Accepted 05 January 2019; Available online 30 April 2019

\begin{abstract}
In the current paper, nanobiocomposite consisting of polylactic acid (biodegradable) (PLA) and nanohydroxyapatite (bioactive) (n-HAP) extracted from bovine bone was fabricated through melt mixing and injection moulding technique for biomedical applications. Partially biogenic nanohydroxyapatite was obtained from bovine bone by hydrothermal method and calcination treatment without using of any chemicals/solvents. Physicomechanical properties of neat-PLA and PLA/n-HAP nanobiocomposite were evaluated using X-ray diffraction (XRD), universal testing machine (UTM) and scanning electron microscopy (SEM). XRD result showed that the intensity of n-HAP peaks increased in the nanobiocomposite as n-HAP-900 loading increased. Tensile strength decreased with increasing the n-HAP-900 loading from 56.78 to $48.25 \mathrm{MPa}$ due to poor interfacial adhesion between neat-PLA and n-HAP. PLA/n-HAP with $1 \%$ loading exhibits tensile strength potential for bone implant application and can be promising biomedical materials for orthopedic applications.
\end{abstract}

Keywords: Bovine bone, Nanohydroxyapatite, Polylactic acid, nanobiocomposite

\section{Introduction}

The number of patients suffering from bone problems such as bone degradation and bone defects besides other related problems develops at an alarming percentage. Research is underway to decrease this figure since most medical procedures such as autografts and allograft are often escorted by specific restrictions [1,2]. Moreover, the problems associated with metallic implants such as stress shielding, non-degradation, poor adhesion, creep, metallic ion release, and problems of biocompatibility with host tissues limits its application in the biomedical field. Attention is consequently focused on the development of biodegradable bioactive composite which are appropriate for bone replacement, and which can provide the expected efficiency [3,4]. Among these, the composites of polylactic acid (PLA) and hydroxyapatite (HAP) gained a lot of consideration. PLA is considerably studied as a synthetic bioabsorbable polymer for potential applications in bone tissue engineering. The advantages of PLA are its bioresorbability, biocompatibility, biodegradability, versatility and ease of manufacturing [1,5] Though, PLA alone often does not have the mechanical strength required for loading application, lack of ability to integrate with the bone 
and discharge acidic degradation products that may have adverse effects on the tissue response [6]. Alternatively, some research has reported that pure polymer implants ordered for bone reconstruction were not practical [7]. So far, many approaches have been studied for the modification of the PLA structure to enhance its physical and mechanical properties [8,9]. The incorporation of the nanoparticles of HAP is one of the effective procedures. n-HAP is the chief mineral component of human bone and consequently has good tissue biocompatibility and bioactivity. PLA/n-HAP nanobiocomposites combine n-HAP bone binding and osteoconductivity with the easy processing assets of PLA. Furthermore, n-HAP can be used to neutralize its acid degradation products, delay the initial degradation of PLA and improve the mechanical performance of the composite. Additionally, the incorporation of the n- HAP into the PLA was found to favor bone tissue growth in implants while the polymer was slowly absorbed into the body without aseptic reactions $[3,10,11]$. Therefore, PLA/n-HAP nanobiocomposites have the prodigious potential for applications in biomedical implant materials due to its good bioactivity, absorption and degradation properties [12-14]. The PLA matrix would adapt the flexibility, ductility and biodegradability of composites and n-HAP would offer osteoconductivity. There are insufficient publications involving injection moulding of PLA/n-HAP nanobiocomposite systems composed of PLA and biogenic n-HAP. Consequently, it would be fascinating and significant to explore the properties of injection molded PLA/n-HAP nanocomposites, which could provide a good groundwork for the fabrication of PLA-based biomedical composite with good inclusive performance [5]. Therefore, injection moulding technology was employed to fabricate PLA/n-HAP nanocomposite in this current research. The aim of this study is to explore the physicomechanical properties of nanobiocomposite composed of PLA and biogenic n-HAP in order to investigate its suitability for loadbearing bone implant applications.

\section{Materials and methods}

The fresh cortical bone of adult bovine ( 2-3 years old) was used as raw material. The bovine bone was purchased from the local market of Malaysia (Parit Raja). Commercial grade polylactic acid (PLA) (Ingeo ${ }^{\text {TM }}$ biopolymer 3052 D), having melt flow index (MFI) value of $14 \mathrm{~g} / 10$ minute and specific gravity of 1.24 , was purchase from Nature Works LLC, USA. Mechanical properties of the PLA-HA composites were evaluated by UTM and effect of n- HAP loading in the PLA matrix was examined by SEM.

\subsection{Extraction of biogenic n-HAP}

Extraction of biogenic n-HAP was carried out according to the method of Bano et al.[15, 16]. In short, bovine bones were cleaned with water and any residues inside the bones were detached manually by a stiletto. Then the bones were cut into small pieces, boiled with water for 2 to 3 hours and washed with distilled water several times. The cleaned bone pieces were sterilized in an autoclave using distilled water for 60 minutes at a high pressure of $0.4 \mathrm{MPa}$ and at a temperature of $129^{\circ} \mathrm{C}$. Finally, the bone pieces were dehydrated in an oven at $80^{\circ} \mathrm{C}$ for 3 days to denaturalize the protein. After that, dry pieces of bovine bone were abridged to small particles by means of the crusher. The crushed bone was subjected to ball milling process for 24 hours at $250 \mathrm{rpm}$. Bovine bone powder was obtained by sieving. The achieved particle size of bone powder used in this study was $25 \mu \mathrm{m}$. The powder samples of $\mathrm{n}$-HAP were calcined in a furnace with 3 hours holding time at a heating and cooling rate of $5^{\circ} \mathrm{C} /$ minute at temperatures of $900^{\circ} \mathrm{C}$ which named as n-HAP-900.

\subsection{Fabrication of PLA/n-HAP nanobiocomposite}

Different formulations of PLA/n-HAP-900 nanobiocomposites samples were fabricated by melt mixing method in two roll mill model PW3000. At first, PLA and n-HAP-900 were dried in a vacuum oven at $60^{\circ} \mathrm{C}$ for 24 hours to eliminate residual moisture prior to the melt processing to prevent its degradation due to hydrolysis during processing as suggested by Chieng et al. [17]. PLA/n-HAP nanobiocomposites were prepared by varying n-HAP-900 loading $(1 \mathrm{wt} \%, 3 \mathrm{wt} \%$ and $5 \mathrm{wt} \%)$. PLA as a matrix and the extracted biogenic n-HAP calcined at $900^{\circ} \mathrm{C}$ were melt-mixed using a two-roll mill machine. Temperature limits of the front and behind rolls are $180^{\circ} \mathrm{C}$ whereas the roller speed was set at $80 \mathrm{rpm}$., n-HAP was fed into the roller carrying the pre-melted PLA, to ensure appropriate mixing and compounding as reported in the literature [18]. Total mixing time was retained constant to 10 minutes for PLA and all the nanobiocomposites to ensure similar thermal history. The collected nanobiocomposites were cooled and cut into smaller pieces using a crusher. The crushed samples were kept in air tight plastic bags for further processed by injection molding. Dumbbell-shaped specimens were produced by injection moulding machine at $190^{\circ} \mathrm{C}$ and $20 \mathrm{MPa}$ for $10 \mathrm{~min}$. and cooled to room temperature at $5 \mathrm{MPa}$.

\section{Characterizations}

X-ray diffraction studies on the n-HAP, neat-PLA and PLA/n-HAP-900 nanobiocomposite were carried out using a high-resolution X-ray diffraction (XRD Model Bruker D8 Advance). The scanning range (2 $\theta$ ) was achieved from $10^{\circ}$ to $80^{\circ}$ in a reflection mode with a step size of $0.02^{\circ}$ per second at room temperature. All patterns were operated at $40 \mathrm{kV}$ and 40mA. The files for n-HAP (Card No. 09-432 was used to identified relative proportions of n-HAP phases present in the nanobiocomposite sample analyzed. The tensile test of PLA/n-HAP-900 nanobiocomposites containing (0wt\%, $1 \mathrm{wt} \%, 3 \mathrm{wt} \%$ and $5 \mathrm{wt} \%$ ) of $\mathrm{n}$-HAP was performed on a universal testing machine (UTM) (AGS-J Shimadzu) at room 
temperature in accordance to ISO 527-1B (1993) with effective dimensions of $75 \mathrm{~mm} \times 12.3 \mathrm{~mm} \times 2 \mathrm{~mm}$ and a $25 \mathrm{~mm}$ gauge length. Tensile strength was measured with a crosshead speed of $5 \mathrm{~mm} / \mathrm{min}$. and a load cell of $5 \mathrm{kN}$ on dry samples. Data for tensile test were recorded. At least 5 specimens were used for each set of nanobiocomposite and average results were taken as the resultant value. A scanning electron microscope (SEM) (HITACHI SU1510) operating at $15 \mathrm{kV}$ was used to visualize fractures surfaces morphology of the neat-PLA and PLA/n-HAP-900 nanobiocomposites having different $w t \%$ of $n$-HAP-900 to check the effect of $n$-HAP loading in the PLA matrix. All samples were coated with a thin layer of Gold/Palladium $(\mathrm{Au} / \mathrm{Pd})$ before examining. From the SEM analysis of neat PLA and PLA/n-HAP-900 nanobiocomposites, information about the microstructure and interaction between n-HAP900 filler and PLA matrix of a composite was obtained.

\section{Results and discussion}

\subsection{XRD analysis}

Fig. 1 shows the relevant XRD patterns of the neat-PLA, n-HAP-900 and PLA/n-HAP-900 nanobiocomposites having different n-HAP-900 loadings. The XRD patterns exhibit the characteristics peaks of the PLA and n-HAP phase except for the differences in peak absolute intensity of the diffraction patterns. These sharp peaks observed in Fig. 1. designate nanocrystalline nature of the extracted n-HAP samples. The prominent diffraction peaks at $2 \theta$ values of $25.80^{\circ}, 28.40^{\circ}, 31.48^{\circ}, 32.68^{\circ}, 34.89^{\circ}$ and $40.04^{\circ}$ corresponding to the (002), (210), (211), (300), (202) and,(310) miller planes for n-HAP-900 and were matched with the JCPDS card 9-432 of HAP [19].

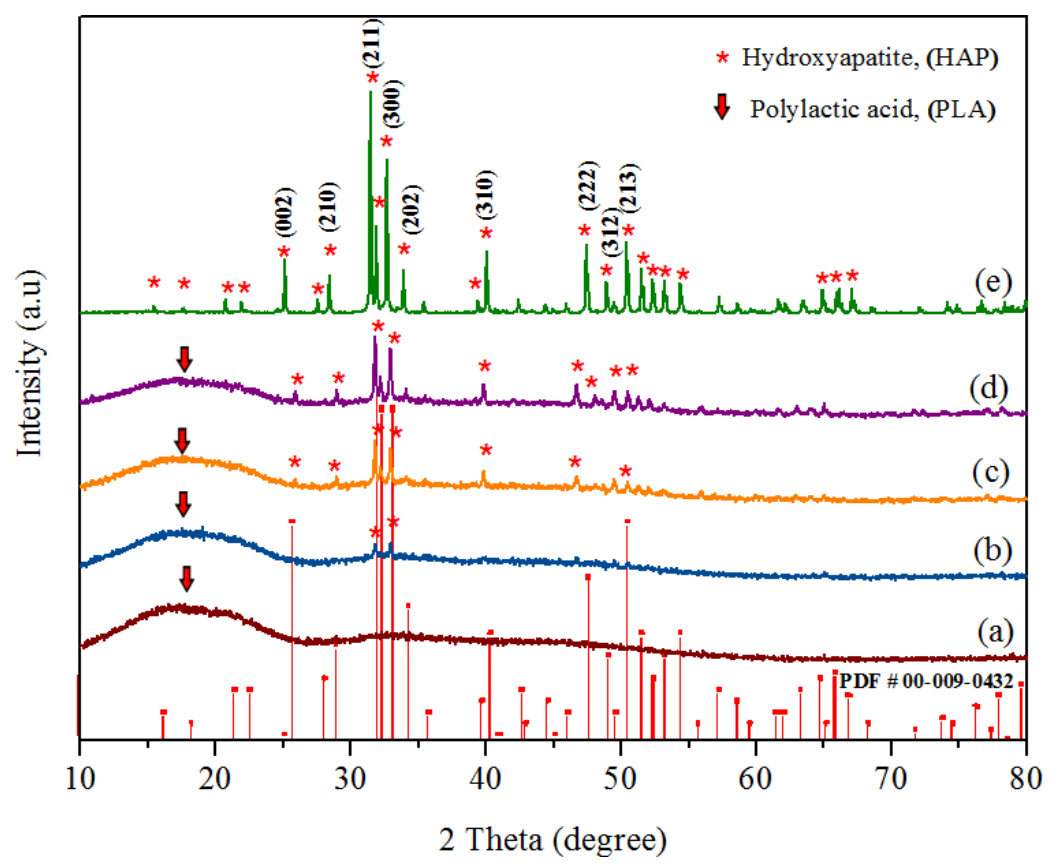

Fig. 1 - XRD pattern of n-HAP-900, neat-PLA and PLA/n-HAP-900 nanobiocomposites with different wt $\%$ of nHAP-900 loading (a) neat PLA (b), PLA/1\% n-HAP-900, (c) PLA/3\%n-HAP-900, (d) PLA/5\% n-HAP-900 and (e) n-HAP-900

The XRD pattern of neat-PLA (Fig. 1 (a)) shows a characteristic of a broad peak at $2 \theta$ values of $16.80^{\circ}$. This wide diffraction peak of the nanobiocomposite can be attributed to amorphous PLA as reported in the literature [20]. The intensity reduction of XRD pattern of neat-PLA peaks in the nanobiocomposite indicates that the interfacial bonding between n-HAP and PLA changes neat-PLA crystal structure and decreases its crystallinity [21]. Because PLA is hydrophobic and n-HAP is hydrophilic therefore hydrogen bonding is formed between carbonyl groups from PLA and hydroxyl groups from n-HAP leading to the formation of nanobiocomposite.

\subsection{Tensile strength}

In this study, all PLA/n-HAP-900 nanobiocomposites showed similar inclinations. With increasing n-HAP loading, tensile strength decreased. Higher filler loading has changed the nanobiocomposite from ductile to brittle as stated by Gay and coworkers [22]. The tensile strength result of neat PLA and PLA/n-HAP-900 nanobiocomposite at different wt $\%$ of n-HAP (1-5wt\%) is presented in Fig. 2. 


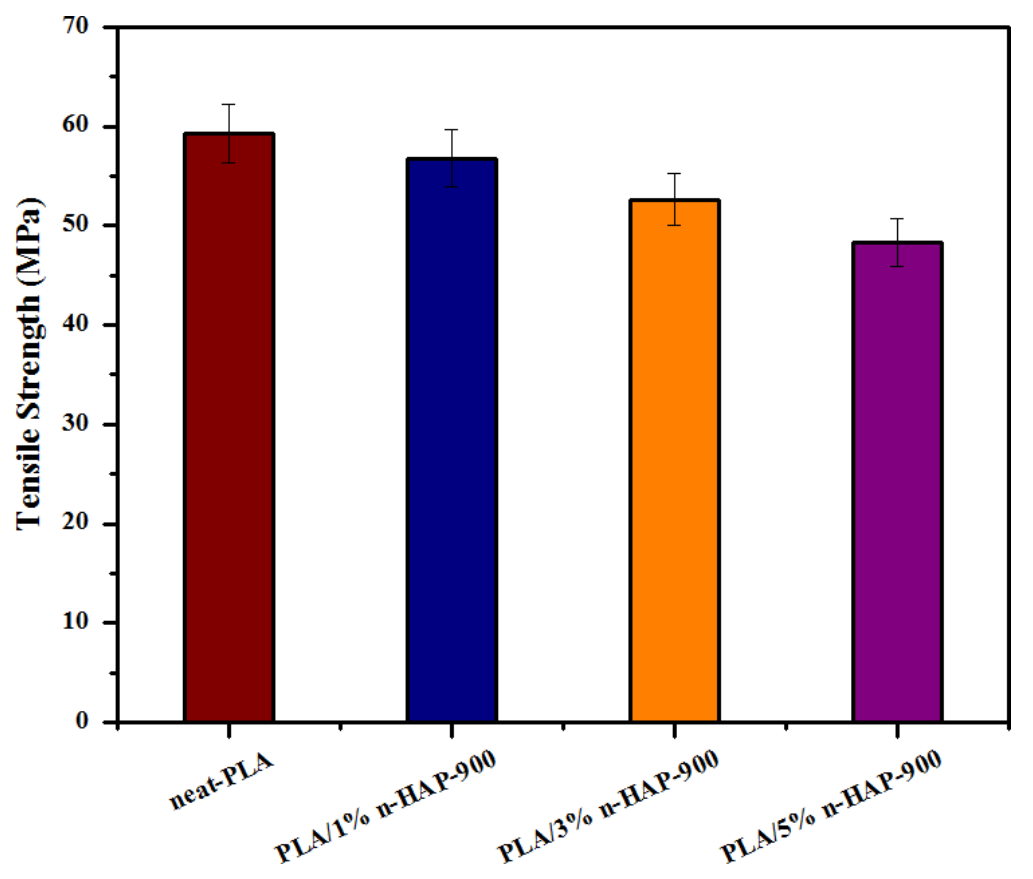

Fig. 2 - Tensile strength of neat PLA and PLA/n-HAP-900 nanobiocomposites with different n-HAP loading

It is observed that the tensile strength is decreased when the n-HAP loading is increased. This indicated the weak interaction between the n-HAP and neat-PLA at higher filler loading. The tensile strength for pure PLA is 59.23 MPa. This value had reduced to $56.78 \mathrm{MPa}, 52.65$ and $48.25 \mathrm{MPa}$ after incorporation of $1 \mathrm{wt} \%$, 3wt $\%$ and $5 \mathrm{wt} \%$ of n-HAP respectively, into the PLA. At $1 \mathrm{wt} \% \mathrm{n}$-HAP content, the tensile strength of the nanobiocomposite was $56.78 \mathrm{MPa}$ which is a reduction of about $2.59 \%$ in respect to neat-PLA. Further increase in n-HAP loading to 3 wt $\%$, the tensile strength was $52.65 \mathrm{MPa}$ which is a reduction of about $11.10 \%$ in respect to neat PLA. Additionally, with respect to the $1 \%$ n-HAP, the tensile strength of the $5 \mathrm{wt} \% \mathrm{n}$-HAP decreased by $15.0216 \%$. This decrease can be associated with the low strength of n-HAP as reported in the literature [23], possibly due to the elimination of organic components during extraction. Similarly, it was stated in the literature that the mechanical properties of PLA/HAP nanobiocomposites are greatly dependent on the dispersion of n-HAP into the PLA matrix [24].

Consequently, distribution issues may be accountable for the experiential decrease in tensile strength as the nHAP loading upsurges. The osteoconductive n-HAP filler intermingles in two ways; chemically due to chemical interactions between the polar groups of n-HAP $(-\mathrm{OH})$ and PLA $(-\mathrm{OH},-\mathrm{COO}$ and $-\mathrm{C}=\mathrm{O})$ and additionally, physically as the particles are highly porous, increasing the surface tension (due to surface roughness (dispersive component)) as reported by Zhang et al. [25]. Both interactions make the interfacial adhesion between the neat-PLA and the n-HAP increase and, subsequently, the rigidity of the fabricated nanobiocomposites [26]. It is worth noting that, although the tensile strength decreased upon an increase in n-HAP loading, the results obtained for PLA/n-HAP-900 nanobiocomposites with different $\mathrm{n}-\mathrm{HAP}$ loading are able to mimic the tensile strength of cortical bones (50-60MPa) [27].

\subsection{Scanning Electron Microscopy (SEM)}

Fig.3 illustrates the SEM fractography of the tensile fractured surface of neat-PLA and PLA/n-HAP-900 nanobiocomposites with $1 \mathrm{wt} \%, 3 \mathrm{wt} \%$ and $5 \mathrm{wt} \% \mathrm{n}-\mathrm{HAP}-900$ loading at 500X magnification. It can be seen that neatPLA shows a comparatively smooth fracture with crinkles of wavy lines, demonstrating characteristic brittle fracture behavior. There is no n-HAP-900 loading seen in the image confirming the neat-PLA (Fig. 3 (a)). Related to the neatPLA, the fracture surfaces of PLA/n-HAP-900 nanobiocomposites exhibited significantly different fractured surfaces whereby much rougher fracture surfaces are observed as the loading of n-HAP-900 increased (Fig. 3 (b-d)). Based on the observation, it is evidenced that n-HAP-900 particles were dispersed homogeneously and individually in the PLA/nHAP-900 nanobiocomposite with 1 to $5 \mathrm{wt} \%$ n-HAP-900 loading. In some part of the nanobiocomposites agglomeration of particles can be found and some parts were left with voids due to the detachment of n-HAP-900 filler. Upon addition of $3 \mathrm{wt} \%$ n-HAP-900, presence of voids can be detected in Fig. 3 (c). However, from Fig. 3 (d), it can be observed that the n-HAP-900 particles were agglomerated at some places when the n-HAP-900 loading was increased $(5 \mathrm{wt} \%)$. 

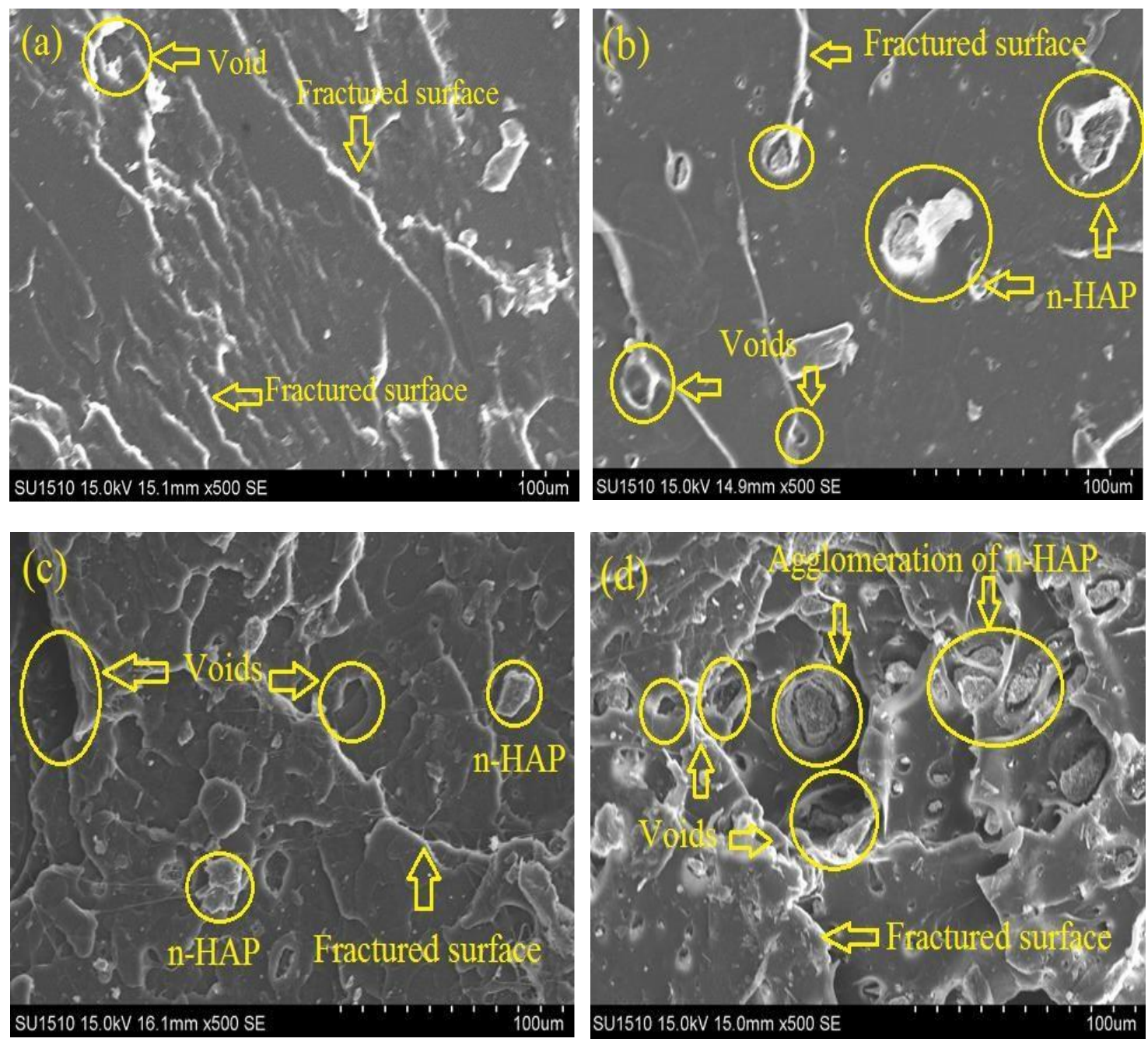

\section{Fig. 3 - SEM fractography of fractured surface of PLA/n-HAP-900 nanobiocomposites with different wt $\%$ of n- HAP-900 loading (a) neat-PLA (b), PLA/1\%n-HAP-900, (c) PLA/3\% n-HAP-900, (d) PLA/5\% n-HAP-900}

Furthermore, the poor interfacial adhesion of the PLA matrix with the n-HAP-900 at higher loading (3 wt $\%$ and 5 wt $\%$ ) is noticed in Fig. 3 (c and d). These observations are in line with the decrease in mechanical properties detected with the addition of $5 \mathrm{wt} \%$ of n-HAP-900 loading. The voids adjacent to n-HAP-900 particles proposed that the interface between neat-PLA and n-HAP filler needed to be enhanced, as the voids may lead to early failure of the nanobiocomposite, consequently restraining the mechanical properties of neat-PLA based nanobiocomposite. Fundamentally, a good distribution of the inorganic filler in the PLA matrix is essential during melt compounding [28]. Therefore, while melt compounding produced a good distribution of the n-HAP-900 filler in the PLA matrix, the degradation of PLA was another concern for mechanical improvement [29].

\section{Conclusion}

Biogenic n-HAP has been successfully extracted from bovine bone and successfully used to prepare PLA/n-HAP900 nanobiocomposite via melt compounding cum injection moulding. XRD result showed that the intensity of $n$-HAP peaks increased in the biocomposite as n-HAP-900 loading increased. These changes indicate that n-HAP crystal structure changes after biocomposite formation. Tensile strength decreased with increasing the n-HAP-900 loading from 56.78 to $48.25 \mathrm{MPa}$ due to poor interfacial adhesion between neat-PLA and n-HAP. SEM fractography evidenced that the n-HAP-900 particles were agglomerated at some places when the n-HAP-900 loading was increased (5 wt $\%$ ) and a transition from ductile surface to brittle surface Therefore it is determined that $1 \mathrm{wt} \%$ of $\mathrm{n}$-HAP loading in to PLA matrix gives the tensile strength value of 56.78 MPa which also mimic the tensile strength of cortical bones (50$60 \mathrm{MPa}$ ). Thus, it is concluded that PLA/n-HAP-900 nanobiocomposite can be promising biomedical materials for orthopedic applications. 


\section{Acknowledgement}

This study is partially supported by the Centre for Graduate Studies (CGS), Universiti Tun Hussein Onn Malaysia from the UTHM scholarship (UTHM/PS/500-14/8).

\section{References}

[1] Hickey, D. J., Ercan, B., Sun, L., Webster, T. J. (2015). Adding MgO nanoparticles to hydroxyapatite-PLLA nanocomposites for improved bone tissue engineering applications. Acta Biomaterialia, 14, 175-84.

[2] Narayanan, G., Vernekar, V. N., Kuyinu, E. L., Laurencin, C. T. (2016). Poly (lactic acid)-based biomaterials for orthopaedic regenerative engineering. Advanced Drug Delivery Reviews, 107, 247-76.

[3] Zhou, Z., Liu, X., Liu, L., Yi Q. (2009). Fabrication and Properties of Composite Biomaterials Composed of Poly(L-Lactide) and Bovine Bone. Designed Monomers and Polymers, 12, 57-67.

[4] Furukawa, T., Matsusue, Y., Yasunaga, T., Shikinami, Y., Okuno, M., Nakamura, T. (2000). Biodegradation behavior of ultra-high-strength hydroxyapatite/poly (L-lactide) composite rods for internal fixation of bone fractures. Biomaterials, 21, 889-98.

[5] Sulong, A. B., Radzuan, N. A. M., Mamat, M. R., Tharazi, I., Tholibon, D., Dweiri R., et al. (2018). Kenaf Reinforced PLA Composite Thermoforming: A Numerical Simulation. International Journal of Integrated Engineering, 10, 15-20.

[6] Zhou, Z., Liu, L., Liu, Q., Yi, Q., Zhao, Y., Zeng, W., et al. (2013). Biological Assessment of Composite Materials Based on Poly-L-lactide and Bovine Bone. International Journal of Polymeric Materials and Polymeric Biomaterials, 62, 81-4.

[7] Walton, M., Cotton, N. J. (2007). Long-term in vivo degradation of poly-L-lactide (PLLA) in bone. Journal of Biomaterials Applications, 21, 395-411.

[8] Cumkur, E. A., Baouz, T., Yilmazer, U. (2015). Poly(lactic acid)-layered silicate nanocomposites: The effects of modifier and compatibilizer on the morphology and mechanical properties. Journal of Applied Polymer Science, $132,1-12$.

[9] Clasen, S. H., Müller, C. M. O., Pires, A. T. N. (2015). Maleic Anhydride as a Compatibilizer and Plasticizer in TPS/PLA Blends. Journal of the Brazilian Chemical Society, 26, 1583-90.

[10] Zhihua, Z., Jianming, R., Zhongcheng, Z., Jianpeng, Z. (2008). Synthesis and properties of composite biomaterials based on hydroxyapatite and poly(l-lactide). Polymer-Plastics Technology and Engineering, 47, 496-501.

[11] Hasegawa, S., Ishii, S., Tamura, J., Furukawa, T., Neo, M., Matsusue, Y., et al. (2006). A 5-7 year in vivo study of high-strength hydroxyapatite/poly(L-lactide) composite rods for the internal fixation of bone fractures. Biomaterials, 27, 1327-32.

[12] Khan, A., Shah, M. H., Nauman, M., Hakim I., Shahid, G., Niaz P., et al. (2017). Clinical manifestations of patients with Systemic Lupus Erythematosus (SLE) in Khyber Pakhtunkhwa. Journal of the Pakistan Medical Association, 67, 1180-5.

[13] Shen, L., Yang, H., Ying, J., Qiao, F., Peng, M. (2009). Preparation and mechanical properties of carbon fiber reinforced hydroxyapatite/polylactide biocomposites. Journal of Materials Science: Materials in Medicine, 20, 2259-65.

[14] Loo, S. C. J., Moore, T., Banik, B., Alexis, F. (2010). Biomedical Applications of Hydroxyapatite Nanoparticles. Current Pharmaceutical Biotechnology, 11, 333-42.

[15] Bano, N., Jikan, S. S., Basri, H., Adzila, S., Nuhu A. H. (2017). Natural Hydroxyapatite Extracted From Bovine Bone. Journal of Science and Technology, 9, 22-8.

[16] Bano, N., Adzila, S., Jikan, S. S., Basri, H., Kanasan, N. (2019). Extraction of Biological Apatite from Cow Bone at Different Calcination Temperatures: A Comparative Study. Key Engineering Materials, 796, 46-52.

[17] Chieng, B. W., Ibrahim, N. A., Yunus, W. M. Z. W., Hussein, M. Z. (2014). Poly(lactic acid)/poly(ethylene glycol) polymer nanocomposites: Effects of graphene nanoplatelets. Polymers, 6, 93-104.

[18] Feldmann, M., Heim, H. P., Zarges, J. C. (2016). Influence of the process parameters on the mechanical properties of engineering biocomposites using a twin-screw extruder. Composites Part A: Applied Science and Manufacturing, 83, 113-9.

[19] Nejati, E., Mirzadeh, H., Zandi, M. (2008). Synthesis and characterization of nano-hydroxyapatite rods/poly(1lactide acid) composite scaffolds for bone tissue engineering. Composites Part A: Applied Science and Manufacturing, 39, 1589-96.

[20] Abdal-Hay, A., Sheikh, F. A., Lim, J. K. (2013). Air jet spinning of hydroxyapatite/poly(lactic acid) hybrid nanocomposite membrane mats for bone tissue engineering. Colloids and Surfaces B: Biointerfaces, 102, 63543.

[21] Liao, S. S., Cui, F. Z., Zhang, W., Feng, Q. L. (2004). Hierarchically Biomimetic Bone Scaffold Materials: Nano-HA/Collagen/PLA Composite. Journal of Biomedical Materials Research - Part B Applied Biomaterials, 69, 158-65.

[22] Gay, S., Arostegui, S., Lemaitre, J. (2009). Preparation and characterization of dense nanohydroxyapatite/PLLA 
composites. Materials Science and Engineering C, 29, 172-7.

[23] Cucuruz, A. T., Andronescu, E., Ficai, A., Ilie, A., Iordache, F. (2016). Synthesis and characterization of new composite materials based on poly(methacrylic acid) and hydroxyapatite with applications in dentistry. International Journal of Pharmaceutics, 510, 516-23.

[24] Liuyun, J., Chengdong, X., Lixin, J., Dongliang, C., Qing, L. (2013). Effect of n-HA content on the isothermal crystallization, morphology and mechanical property of n-HA/PLGA composites. Materials Research Bulletin, $48,1233-8$

[25] Zhang, H., Lu, X., Leng, Y., Fang, L., Qu, S., Feng, B., et al. (2009). Molecular dynamics simulations on the interaction between polymers and hydroxyapatite with and without coupling agents. Acta Biomaterialia, 5, 1169-81.

[26] Ferri, J., Jordá, J., Montanes, N., Fenollar, O., Balart, R. (2017). Manufacturing and characterization of poly(lactic acid) composite with hydroxyapatite. Journal of Thermoplastic Composite Materials. 31,865-81.

[27] Roeder, R. K., Converse, G. L., Kane, R. J., Yue, W. (2008). Hydroxyapatite-reinforced polymer biocomposites for synthetic bone substitutes. Jom, 60, 38-45.

[28] Zhou, H., Lawrence, J. G., Bhaduri, S. B. (2012). Fabrication aspects of PLA-CaP/PLGA-CaP composites for orthopedic applications: A review. Acta Biomaterialia, 8, 1999-2016.

[29] Liu, X., Wang, T., Chow, L. C., Yang, M., Mitchell, J. W. (2014). Effects of inorganic fillers on the thermal and mechanical properties of poly(lactic acid). International Journal of Polymer Science, 827028. 